\title{
Benigne und maligne Gallenwegerkrankungen
}

\author{
J. F. Riemann, Ludwigshafen R. Bittner, Stuttgart
}

Gallenwegerkrankungen sind ein typisches Beispiel dafür, daß mit modernen diagnostischen Verfahren in der Regel eine präzise Diagnose gestellt und damit häufig unmittelbar im Anschluß eine Therapie durchgeführt werden kann. Gleichzeitig repräsentieren sie Erkrankungen, bei denen das interdisziplinäre Gespräch zwischen Chirurgen und Gastroenterologen unter Einschaltung der Pathologen eine wesentliche Voraussetzung für eine effiziente Behandlungsstrategie ist. Interventionelle endoskopische Verfahren können z. B. beim Gallengangsteinleiden mit großem Erfolg eingesetzt werden, ohne daß ein operativer Eingriff nötig wird. Auf der anderen Seite ist die präzise Lokalisationsdiagnostik beim Hepatikusgabeltumor eine entscheidende Voraussetzung für ein maßgeschneidertes operatives Therapiekonzept. In dieser Situation stehen nicht nur die modernen bildgebenden Verfahren wie Magnetresonanz-Cholangiopankreatikographie und gegebenenfalls intraduktale Sonographie, sondern auch bioptisch zytologische Maßnahmen zur Verfügung.

Einen hohen Stellenwert im interdisziplinären Therapiekonzept haben Stenosierungen des intrapankreatischen Anteils des Ductus choledochus bei der chronisch rezidivierenden Pankreatitis. Hier ist eine ausgesprochen sensible Abstimmung zwischen Gastroenterologen und Chirurgen gefordert.

Während unverändert die periampullären Karzinome überwiegend eine Domäne der Chirurgie sind, konnten besondere Fortschritte in der palliativen Behandlung maligner Gallenwegobstruktionen erzielt werden. Durch geeignete Drainagemaßnahmen lassen sich hiermit Patienten bei guter Lebensqualität versorgen. Allerdings gilt es im Einzelfall stets sorgfältig abzuwägen, welcher Patient einer endoskopischen interventionellen, einer perkutan transhepatischen Drainage oder einem chirurgischen Bypassverfahren zugeführt werden soll.

Das Spektrum der diagnostischen und therapeutischen Möglichkeiten ist äußerst umfangreich; prospektive Studien müssen in $\mathrm{Zu}$ kunft auch unter ökonomischen Gesichtspunkten und im Hinblick auf Ergebnisqualität zeigen, mit welchen Maßnahmen in welcher Situation individuell für jeden Patienten die besten Erfolgsaussichten für die Behandlung bestehen. Bereits jetzt kann jedoch postuliert werden, daß die enge Kooperation von Gastroenterologen, Chirurgen und Radiologen unverzichtbar ist.

\section{KARGER}

(C) 1999 S. Karger GmbH, Freiburg

Fax +497614520714

E-mailkargergmbh@aol.com

www.karger.com
Prof. Dr. J. R. Riemann

Medizinische Klinik C

Klinikum der Stadt Ludwigshafen $\mathrm{gGmbH}$

Bremserstraße 79

D-67063 Ludwigshafen 